\title{
EFFECT OF CHAOTIC ORBITS ON DYNAMICAL FRICTION
}

\author{
SOFÍA A. CORA* , M. MARCELA VERGNE and JUAN C. MUZZIO \\ Facultad de Ciencias Astronómicas y Geofísicas, Universidad Nacional de La Plata, \\ Paseo del Bosque, 1900 La Plata, Argentina
}

\begin{abstract}
When a body moves through a medium of smaller particles, it suffers a deceleration due to dynamical friction (Chandrasekhar 1943). Dynamical friction is inversely proportional to the relaxation time, which can be defined as the time needed for the orbits to experiment an energy exchange of the order of their initial energies, as a result of the perturbations produced by stellar encounters. Chaotic orbits, present in non-integrable systems, have exponential sensitivity to perturbations, a feature that makes them to relax in a time much shorter than regular ones, which suggests that dynamical friction would increase in the presence of chaotic orbits (Pfenniger 1986). We present preliminary results of numerical experiments used to check this idea, investigating the orbital decay, caused by dynamical friction, of a rigid satellite which moves within a larger stellar system (a galaxy) whose potential is non-integrable. Triaxial models with similar density distributions but different percentages of chaotic orbits are considered. This last quantity depends on the central concentration of the models. If the potential corresponds to triaxial mass models with smooth cores, the regular orbits have shapes that can be identified with one of the four families of regular orbits in Stäckel potentials (box and three types of tubes). Chaotic orbits behave very much like regular orbits for hundreds of oscillations at least. In this case, the galaxy is represented by the triaxial generalization of the $\gamma$-models with $\gamma=0$ (Merritt \& Fridman 1996). However, the situation is very different in triaxial models with divergent central densities (cusps) or black holes, a feature that is in agreement with the observations. While the tube orbits are not strongly affected by central divergencies, the boxlike orbits are often rendered chaotic (Gerhard \& Binney 1985). The timescale in which the chaos manifests itself in the orbital motion is short compared to a Hubble time. In this models, the compact object is taken as a Plummer sphere.
\end{abstract}

The percentage of regular and chaotic orbits in a given model is obtained computing the six Liapunov exponents for each orbit of the particles that make up the galaxy.

The initial conditions of the particles that make up the galaxy $(N=10,000)$ are obtained generating them in the corresponding spherical model with isotropic velocity distribution, and integrating them for a few dynamical times in the potential corresponding to the triaxial model with core (axis ratios $c / a=0.5, b / a=0.79$ ) and black hole (mass $m_{h}=0.02 M$ and softening parameter $\varepsilon_{h}=0.08$ ); the adopted units are such that the gravitational constant $G$, the total mass $M$ and the $\mathrm{x}$-axis scale length $a$ are unity.

*e-mail: sacora@fcaglp.fcaglp.unlp.edu.ar 
The satellite is modelled by a softened point mass, with mass $m_{s}=0.01 \mathrm{M}$ and softening parameter $\varepsilon_{s}=0.1$. Its initial position corresponds to a distance slightly larger than the radius containing the half mass of the galaxy. Its velocity is such that it would move on a circular orbit in the spherical model.

To integrate the equations of motion, a non-self-consistent code is used: the particles move in the fixed potential that represents the galaxy and do not interact with each other; the satellite interacts with the particles in order to simulate the dynamical friction process. This method allows one to know whether an orbit is regular or chaotic in a given potential.

The satellite decay rates in models with a black hole are greater in some cases, and smaller in others, than in models without it. It is possible, however, that to affect the orbital decay, chaotic orbits should have a Liapunov time $\left(T_{\text {lia }}\right)$ shorter than the time required by the satellite to reach the center of the galaxy $\left(T_{d e c} \simeq 18\right.$ crossing times). The lack of a clear behaviour of the satellite may be attributed to the fact that the perturbation produced by the black hole is not strong enough to produce a great difference in the percentages of chaotic orbits with $T_{l i a}<T_{d e c}$, in the models with and without black hole (25\% and $16 \%$ the total number of particles of the system, respectively). This analysis will be repeated with other galaxy models that present larger differences in their percentages of chaotic orbits, in order to elucidate how the presence of those orbits affects the orbital decay of the satellite.

We are grateful to D. Pfenniger for kindly making his code available to us. This work was supported through grants from CONICET.

\section{References}

Chandrasekhar, S.: 1943, ApJ, 97, 255

Gerhard, O. E., \& Binney, J. J.: 1985, MNRAS, 216, 467

Merritt, D., \& Fridman, T.: 1996, ApJ, 460, 136

Pfenniger, D.: 1986, A\&A, 165, 74 